\title{
Esquemas de alimentación para larvicultura de pejelagarto (Atractosteus tropicus Gill): crecimiento, supervivencia y canibalismo
}

\section{Evaluation of feeding strategies in tropical gar (Atractosteus tropicus Gill) larvae: growth, survival and cannibalism}

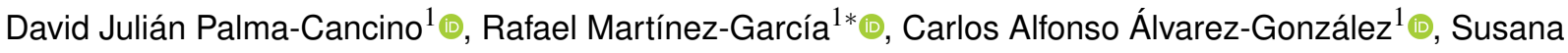

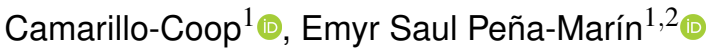 \\ ${ }^{1}$ División Académica de Ciencias Biológicas de la Universidad Juárez Autónoma de Tabasco, Carretera Villahermosa-Cárdenas Km 0.5 \\ $\mathrm{s} / \mathrm{n}$. Entronque a Bosques de Saloya CP. 86150. Villahermosa, Tabasco, México. \\ ${ }^{2}$ Cátedra, Consejo Nacional de Ciencia y Tecnología, Universidad Juárez Autónoma de Tabasco, Ciudad de México, México. \\ *Autor de correspondencia: biologomartinez@hotmail.com
}

Artículo científico recibido: 16 de enero de 2019 aceptado: 25 de febrero de 2019

RESUMEN. El diseño de dietas con base en la fisiología digestiva, así como un adecuado esquema de alimentación, permiten la disminución del canibalismo y el incremento de la supervivencia en la etapa larvaria de peces. Por lo anterior, el objetivo fue evaluar el crecimiento, supervivencia y canibalismo en la larvicultura del pejelagarto (Atractosteus tropicus Gill) bajo cuatro esquemas de alimentación: CT (co-alimentación con nauplio de Artemia por 10 días y dieta comercial de trucha), T1 (alimentación con la dieta comercial de trucha desde la apertura de la boca), T2 (co-alimentación con nauplio de Artemia y dieta diseñada para larvas de $A$. tropicus por 10 días) y T3 (alimentación con la dieta diseñada para $A$. tropicus desde la apertura de la boca), así como determinar la correlación entre el canibalismo y la supervivencia mediante un modelo de regresión lineal. Los mejores crecimientos y supervivencias se obtuvieron en los tratamientos T2 y CT. En todos los tratamientos se presentó canibalismo, siendo más alto cuando no se co-alimentó con nauplio de Artemia (T1 y T3). Se encontró una fuerte correlación $(r=-0.88, p<0.05)$ entre la supervivencia y el canibalismo, lo que demuestra que el canibalismo tiene efecto sobre la supervivencia. El tratamiento T2 representa la mejor alternativa para la larvicultura de $A$. tropicus, con mayor crecimiento y menor canibalismo.

Palabras clave: Canibalismo, co-alimentación, larvicultura, modelo de regresión lineal, pejelagarto.

ABSTRACT. The design of specific feed based on digestive physiology, and an adequate feeding schedule, are important to reduce cannibalism and increase survival rate in larvae fish. The first objective of this study was evaluating growth, survival and cannibalism during tropical gar (Atractosteus tropicus Gill) larviculture, using four feeding schedules: CT (10 day co-feeding of Artemia nauplii and commercial trout feed), T1 (commercial trout feed), T2 (10 day co-feeding of Artemia nauplii and $A$. tropicus larvae designed feed) and T3 ( $A$. tropicus larvae designed feed). The second objective was to determinate correlation between survival and cannibalism, using a linear regression model. We observed better growth and survival in the co-feeding treatments CT and T2. Cannibalism was observed in all treatments, with higher presence in T1 and T3 (treatments without Artemia nauplii co-feeding). It was observed a strong correlation between survival and cannibalism $(r=$ $-0.88, p<0.05$ ), demonstrating that cannibalism has a considerable effect on survival rate. We concluded that T2 represent the best alternative for $A$. tropicus feeding during larviculture, since they present better growth and less cannibalism.

Key words: Cannibalism, larviculture, linear regression model, co-fedding, tropical gar.

\section{INTRODUCCIÓN}

La acuicultura en el sureste de México ha tenido un crecimiento constante en los últimos años, debido a la alta presencia de recursos hídricos en la región. El aprovechamiento de especies nativas dulceacuícolas para diversificación del mercado regional ha generado la necesidad de estudiar el poten- 
cial acuícola de muchas especies endémicas (Saenz de Rodrigáñez et al. 2018). Una de las especies más estudiadas para su producción en cautiverio es el pejelagarto (Atractosteus tropicus Gill), que en estados como Tabasco y Chiapas es muy consumida, teniendo incluso valor cultural (Márquez-Couturier y Vázquez-Navarrete 2015).

Los estudios sobre la biología, reproducción y genética de $A$. tropicus en los últimos 30 años han permito conocer aspectos de su cultivo (AriasRodríguez et al. 2009, Márquez-Couturier y VázquezNavarrete 2015). Pero aún existen problemáticas que evitan un dominio total de las técnicas de su producción. Por ejemplo dentro de la larvicultura de la especie los problemas más importantes son el canibalismo y la falta de dietas comerciales diseñadas para cubrir de forma adecuada los requerimientos nutricionales de la especie, sin embargo, existen estudios que han permitido caracterizar las proteasas digestivas, además de diseñar alimentos experimentales para las etapas tempranas de desarrollo (Guerrero-Zárate et al. 2013, Frías-Quintana et al. 2015).

El canibalismo es un problema significativo sobre todo en especies comerciales carnívoras, lo que causa pérdidas considerables durante su producción (Naumowicz et al. 2017). En este aspecto, el canibalismo entre organismos provenientes de una misma cohorte se presenta principalmente en etapas tempranas del desarrollo y afecta la larvicultura (Król et al. 2014). Una inadecuada densidad de siembra inicial, falta de experiencia del personal, incorrecta alimentación o el desconocimiento del manejo de la especie, son algunas de las principales causas de canibalismo en etapas tempranas de desarrollo (Baras et al. 2003).

En $A$. tropicus al utilizarse una combinación de alimento vivo (Artemia) con dietas comerciales formuladas para trucha se ha observado alta presencia de canibalismo durante los primeros 15 días del cultivo (Frías-Quintana et al. 2010). El canibalismo completo (ingesta total de otro individuo) e incompleto (ingesta parcial o ataque) durante la larvicultura afecta de forma considerable la supervivencia (FríasQuintana et al. 2017). A pesar de existir estrategias para controlar este comportamiento como la separación por tallas (Márquez-Couturier et al. 2015), o el aislamiento (Frías-Quintana et al. 2016), el diseño de dietas específicas es la mejor opción para mitigar este fenómeno (Frías-Quintana et al. 2017).

Durante el larvicultivo de $A$. tropicus, es necesario dejar un periodo de transición con presas vivas o co-alimentación para mejorar la supervivencia y tener un efecto positivo en la producción (EscaleraVázquez et al. 2018). Recientemente se ha incrementado el esfuerzo en la investigación de dietas basadas en la fisiología digestiva larval de $A$. tropicus (Frías-Quintana et al. 2016, Frías-Quintana et al. 2017, Saenz de Rodrigáñez et al. 2018). Pero aún es necesario evaluar el efecto que dichas dietas tienen en el canibalismo los primeros días del cultivo. Por lo anterior, el objetivo de este trabajo fue evaluar distintas estrategias de alimentación durante la larvicultura de $A$. tropicus, midiendo los efectos sobre el crecimiento, la supervivencia y el canibalismo; además de determinar la relación del canibalismo con la supervivencia.

\section{MATERIALES Y MÉTODOS}

\section{Obtención de las larvas}

La obtención de las larvas de $A$. tropicus y el experimento se realizaron en las instalaciones del Laboratorio de Acuicultura Tropical de la División Académica de Ciencias Biológicas de la Universidad Juárez Autónoma de Tabasco (DACBiolUJAT). Las 3600 larvas de A. tropicus empleadas para la realización del experimento se obtuvieron de embriones provenientes de un desove inducido de reproductores ( 1 hembra de $3.5 \mathrm{~kg}$ de peso total y 4 machos con peso promedio de $1.5 \mathrm{~kg}$ ) con $35 \mathrm{mg} \mathrm{kg}^{-1}$ de hormona LHRH-a (Márquez-Couturier et al. 2015). Los reproductores se colocaron en un tanque circular de $2000 \mathrm{~L}$, después del desove y fecundación (16 horas post-inducción) los huevos se retiraron hasta la eclosión (3 días después del desove). Después de la absorción del saco vitelino ( 5 días después de la eclosión), se colocaron las larvas en tanques circulares de plástico con $70 \mathrm{~L}$ de agua, conectados a un sistema de recirculación con reservorio de $1500 \mathrm{~L}$, 
función de filtro biológico y sedimentador de sólidos, además de una bomba de 1 HP (Jacuzzi, JWPA5D230A), y termostato de titanio (PSA ${ }^{M R}$ R9ce371).

\section{Diseño Experimental}

El tratamiento control (CT) se realizó por medio de co-alimentación con nauplio de Artemia salina (Biogrow, PROAQUA ${ }^{\circledR}$ ) y alimento comercial para trucha Silver Cup ${ }^{\mathrm{TM}}$ (45\% de proteína y $16 \%$ de lípidos) durante los primeros 10 días del cultivo, para posteriormente utilizar solo la dieta comercial. Se utilizó la dieta Silver Cup ${ }^{\mathrm{TM}}$ como control, debido a que es el alimento utilizado en los sistemas de cultivo propuestos de pejelagarto (Márquez-Couturier et al. 2015). El tratamiento 1 (T1) consistió en alimentación con dieta Silver Cup ${ }^{\mathrm{TM}}$ para trucha desde el momento de la apertura de la boca (primera alimentación). El tratamiento 2 (T2) consistió en una co-alimentación durante los primeros 10 días con nauplio de Artemia salina y la dieta experimental microparticulada diseñada con base en la fisiología digestiva de la especie (Frías-Quintana et al. 2016); después de los primeros 10 días, se alimentó solo con dieta experimental. El tratamiento 3 (T3), fue la dieta experimental utilizada en T2, desde la apertura de la boca de las larvas. La dieta experimental se elaboró en el Laboratorio de Acuicultura Tropical de la DACBiol-UJAT, su composición se describe en la Tabla 1. El esquema de alimentación utilizado para las larvas en cada tratamiento se ejemplifica en la Figura 1.

La distribución de las larvas y los cuatro tratamientos fue completamente al azar y se replicaron tres veces cada uno, para lo cual se sembraron 300 organismos en cada unidad experimental (tinas ovaladas de plástico con $70 \mathrm{~L}$ de agua conectadas al sistema de recirculación). El experimento duró 45 días, ya que se estima es el tiempo que tardan en llegar a la talla comercial de $80-120 \mathrm{~mm}$ para su venta como pre-juveniles o alevines (Márquez-Couturier et al. 2015).

En cada tratamiento se alimentó a saciedad cinco veces al día durante los primeros 20 días (08:00, 10:15, 12:30, 14:45 y 17:00 horas); del día 21 al 45, las larvas se alimentaron cuatro veces al día
Tabla 1. Preparación de la dieta microparticulada utilizada en T2 y T3.

\begin{tabular}{lc}
\hline Ingredientes & ${\mathrm{g} 100^{-1} \mathrm{~g} \text { de dieta }}$ \\
\hline Harina de pescado $^{a}$ & 40.63 \\
Harina renders (cerdo y pollo) $^{a}$ & 30.07 \\
Almidón de maíz $^{b}$ & 15.4 \\
Aceite de pescado $^{c}$ & 6.9 \\
Lecitina de soya $^{d}$ & 4 \\
Grenetina $^{e}$ & 2 \\
Vitamina C $^{f}$ & 0.5 \\
Pre-mezcla de vitamina y minerales $^{f}$ & 0.5 \\
\hline${ }^{a}$ Proteínas marinas y agropecuarias, Guadalajara, Jalisco, \\
México; $^{b}$ MSA Industrializadora de maíz, Guadalajara, Jalisco, \\
México; ${ }^{c}$ GALMEX Comercializadora de Insumos Agrícolas, \\
${\text { Villahermosa, Tabasco, México; }{ }^{d} \text { Pronat Ultra, Mérida, Yucatán, }}^{\text {México; }}{ }^{e}$ D'Gari Productos alimenticios y dietéticos relámpago, \\
Tlalpan, Cd. de México, México; ${ }^{f}$ ROVIMIX ${ }^{\circledR}$ C-EC (Roche) \\
agente de activo de 35\%, Cd de México, México.
\end{tabular}

a saciedad (08:00, 11:00, 14:00 y 17:00 horas). La cantidad de alimento vivo ( $A$. salina) utilizado en $\mathrm{T} 1 \mathrm{y}$ T2, provino de $0.9 \mathrm{~g}$ de quistes de Artemia por repetición el primer día de cultivo, $1.8 \mathrm{~g}$ el segundo día, 3.6 g el tercer día, $7.2 \mathrm{~g}$ el cuarto día, y $14.1 \mathrm{~g}$ el quinto día. El sexto día se repitió la cantidad de $14.1 \mathrm{~g}$ de quistes, y se disminuyó en la misma proporción que se incrementó hasta que en el día 10 se proporcionó A. salina proveniente de $0.9 \mathrm{~g}$ de quistes. Se estima que por cada gramo de quiste desencapsulado eclosionan 253 individuos de Artemia (Eslava-Eljaiek et al. 2011).

Las heces y alimento no consumido se sifonearon dos veces al día y se realizaron recambios totales de agua cada siete días. La calidad del agua se monitoreó, registrando los datos de temperatura $\left(26.6 \pm 3.7^{\circ} \mathrm{C}\right)$ y oxígeno disuelto $(4.9 \pm 0.4 \mathrm{mg}$ $\mathrm{L}^{-1}$ ) con un oxímetro marca YSITM modelo $55 \mathrm{DO}$, así como $\mathrm{pH}(7.27 \pm 0.23)$ con un potenciómetro marca ADWA modelo AD11.

\section{Crecimiento y supervivencia}

Se realizó una biometría inicial a 30 organismos de forma aleatoria para obtener el promedio de talla $(17.4 \pm 0.6 \mathrm{~mm})$ con un vernier, y el peso $(0.029 \pm 0.002 \mathrm{~g})$ con una balanza analítica OHAUS Explorer 224. Cada 15 días se tomaron de forma aleatoria 30 organismos en cada réplica de cada tratamiento y se registró la longitud total y el peso húmedo. Al final del experimento se tomaron 
Solo Dieta, después de la absorción del saco vitelino (T1 \& T3)

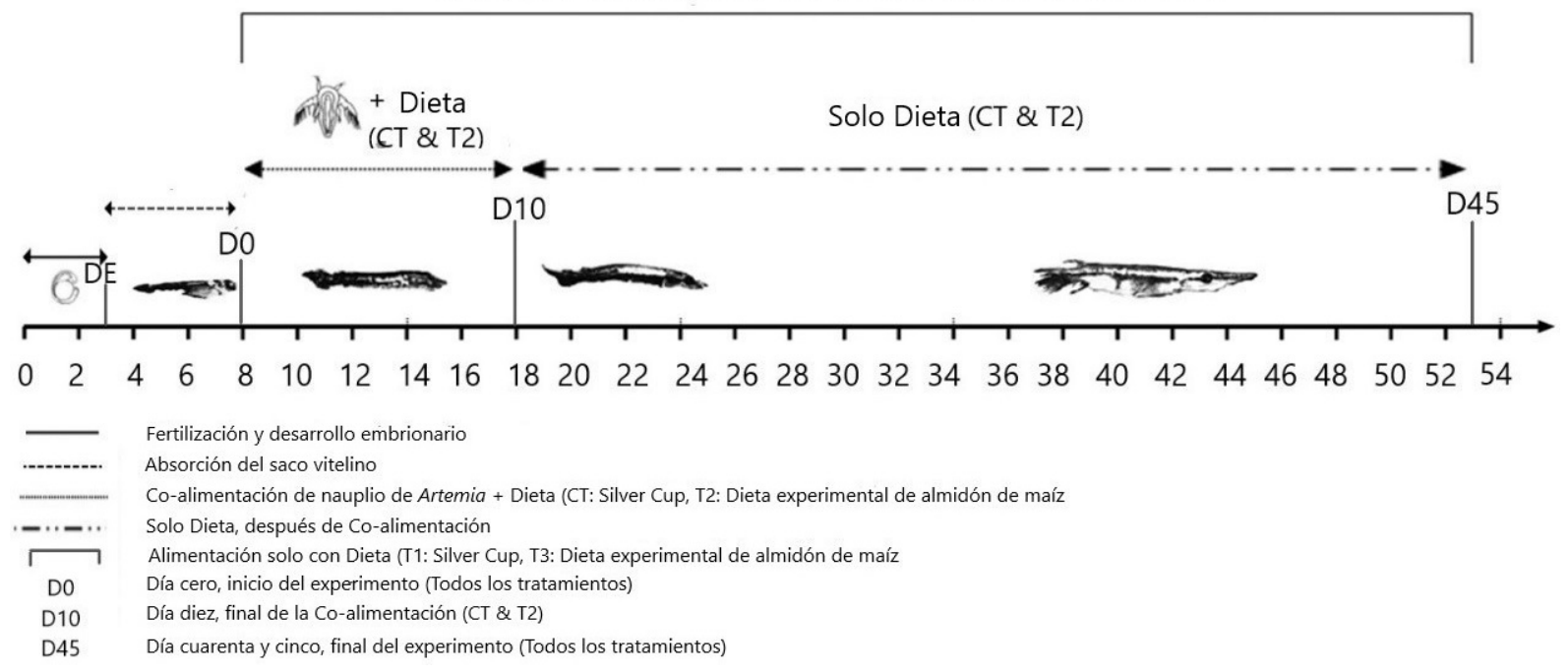

Figura 1. Esquemas de alimentación usados durante el experimento.

todos los organismos vivos en cada réplica de cada tratamiento, para calcular la supervivencia (S) con la siguiente fórmula: Supervivencia $(S)=\left(\frac{N_{f}}{N_{i}}\right) * 100$, donde $\mathrm{N}_{i}$ es el número inicial de organismos y $\mathrm{N}_{f}$ el número final de organismos.

\section{Manejo del canibalismo}

Para evaluar el canibalismo se cuantificaron las larvas que presentaron evidencia de canibalismo completo (deglución total de otro individuo) en cada repetición, y se aislaron en pequeñas jaulas flotantes de plástico dentro de los tanques en que se encontraban, proporcionándoles el mismo tratamiento de alimentación, para que se readaptaran al alimento y posteriormente ser liberados en su respectiva unidad experimental (Frías-Quintana et al. 2016). El canibalismo incompleto (agresividad hacia otro individuo mediante mordedura), fue parcialmente controlado y cuantificado cuando los individuos fueron detectados durante el experimento.

\section{Análisis estadístico}

Para evaluar el crecimiento en talla y peso, a los datos obtenidos se les determinó la homocedasticidad (Levene). A los datos se les realizó un análisis de la varianza de un factor, además de la corrección a posteriori de Bonferroni para encontrar las diferencias entre los tratamientos. Para medir la correlación entre el canibalismo y la supervivencia, se realizó una prueba de regresión lineal simple $\left(R^{2}\right.$ Pearson). Todos los análisis se realizaron con un nivel de significancia de 0.05 , utilizando el software STATGRAPHICS Centurion XVIII.

\section{RESULTADOS}

\section{Crecimiento, supervivencia y canibalismo}

Los resultados de crecimiento mostraron los mayores valores en el tratamiento T2, con peso promedio de $3.37 \pm 1.46 \mathrm{~g}$ y talla de $97.74 \pm 13.07$ $\mathrm{mm}$; siguiéndole los organismos del tratamiento T3 con peso de $2.18 \pm 0.83 \mathrm{~g}$, talla de $84.51 \pm 12.82$ $\mathrm{mm}$; el tratamiento CT tuvo un peso promedio de $2.16 \pm 0.73 \mathrm{~g}$ y talla de $87.5 \pm 9.35 \mathrm{~mm}$; mientras que el tratamiento $\mathrm{T} 1$ tuvo el menor crecimiento con $1.78 \pm 0.45 \mathrm{~g}$ de peso promedio y $80.44 \pm 7.86 \mathrm{~mm}$ de talla promedio. El análisis estadístico determinó que los tratamientos tuvieron diferencias significativas $(p<0.05)$ para ambos valores de crecimiento (Figura 2a, b). El test de Bonferroni determinó que el tratamiento T2 es estadísticamente diferente $(p<$ 0.05 ) a los tratamientos CT, T1 y T3 para peso y lon- 


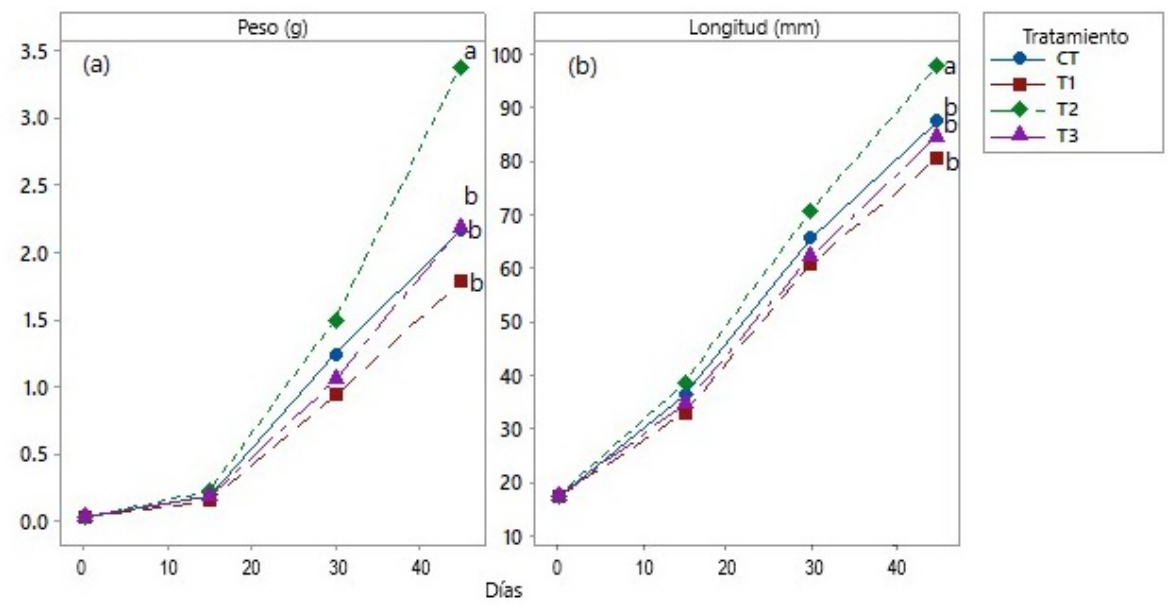

Figura 2. Peso húmedo promedio ( larva $^{-1}, \mathrm{n}=300$ ) (a) longitud promedio total ( $\mathrm{mm} \mathrm{larva}^{-1}, \mathrm{n}=300$ ) (b) en larvas de Atractosteus tropicus, observadas durante el experimento.

gitud total. El mejor porcentaje de supervivencia se observó en los organismos del tratamiento T2 con $32.33 \%$, seguido del tratamiento CT con $15.56 \%$, el T3 con 5.56\%, y el T1 logrando solo una supervivencia del $1 \%$ (Figura 3).

En todos los tratamientos se observó canibalismo durante el cultivo (Figura 3). El total de organismos separados tras observarse comportamiento caníbal para cada tratamiento fue de 89 (9.9\%) para el tratamiento CT, 204 en el T1 (22.67\%), 49 en el T2 (5.44\%), y 40 en T3 (13.55\%); este comportamiento caníbal se observó principalmente entre los 5 y 20 días de inicio del experimento. El porcentaje de canibalismo por tratamiento fue significativamente menor $(p<0.05)$ en el tratamiento T2 al compararlo con los demás tratamientos. Mientras que en el tratamiento T1, se observó una mortalidad de los organismos caníbales, ya que algunos fueron encontrados con otro organismo a medio devorar o al separarlos, murieron uno o dos días después; esto también se observó en los demás tratamientos, pero no más de dos casos. Por último, el canibalismo incompleto se comenzó a observar después del día 16 de cultivo y las víctimas de este comportamiento murieron poco después del ataque.

El análisis de regresión lineal indica que hay relación estadísticamente significativa entre la supervivencia y el canibalismo $(p<0.05)$; el estadístico $R^{2}$ indica que el modelo ajustado explica en un $77.38 \%$ la variabilidad en la supervivencia. Mientras que el coeficiente de correlación $(r=-0.88, p<0.05)$, indica una moderadamente fuerte relación entre las variables (Figura 4).

\section{DISCUSIÓN}

El análisis estadístico de los promedios de las variables longitud total, peso húmedo y supervivencia indicó diferencias entre los tratamientos. El tratamiento T2 presentó el mayor crecimiento y supervivencia $(p<0.05)$ con respecto a los tratamientos CT, T1 y T3, lo que sugiere que es la mejor alternativa de las estrategias evaluadas para la alimentación durante la larvicultura de $A$. tropicus. La longitud total promedio después de 45 días de cultivo, mostró que las larvas de los tratamientos CT, T2 y T3 alcanzaron el tamaño de comercialización para venta de alevines o pre-juveniles dentro de los tiempos reportados por Márquez-Couturier et al. (2015), con crecimientos recomendados de 80 - $120 \mathrm{~mm}$ para pasar a la etapa de alevinaje de la especie.

El crecimiento observado en los tratamientos CT, T2 y T3, resultó similar al obtenido por FríasQuintana et al. (2016), pero en relación a la longitud total se obtuvieron mejores promedios (10 - 40 $\mathrm{mm}$ superiores) en el presente estudio, aunque esto 


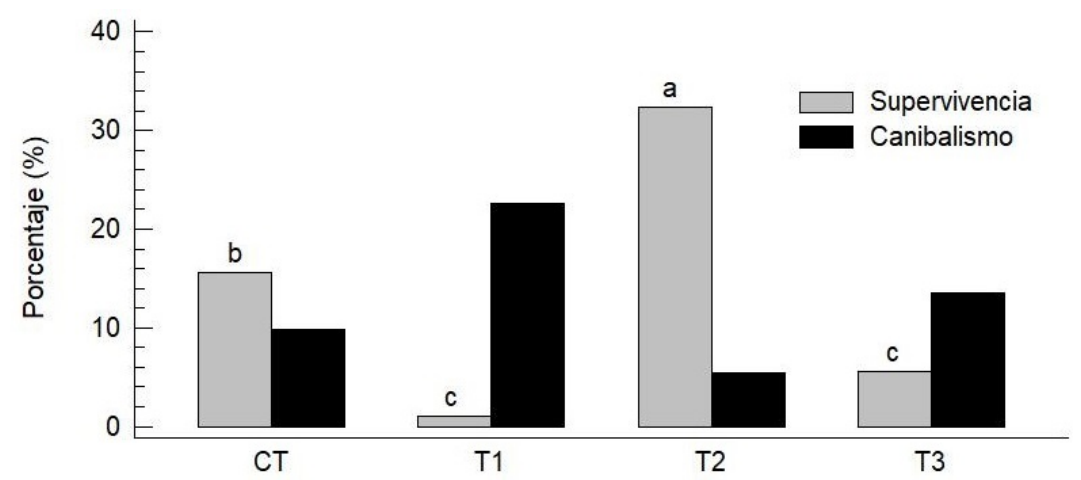

Figura 3. Porcentaje de supervivencia y canibalismo observado en larvas de Atractosteus tropicus alimentados con cuatro tratamientos diferentes. Letras diferentes indican diferencias significativas $(p<0.05)$.

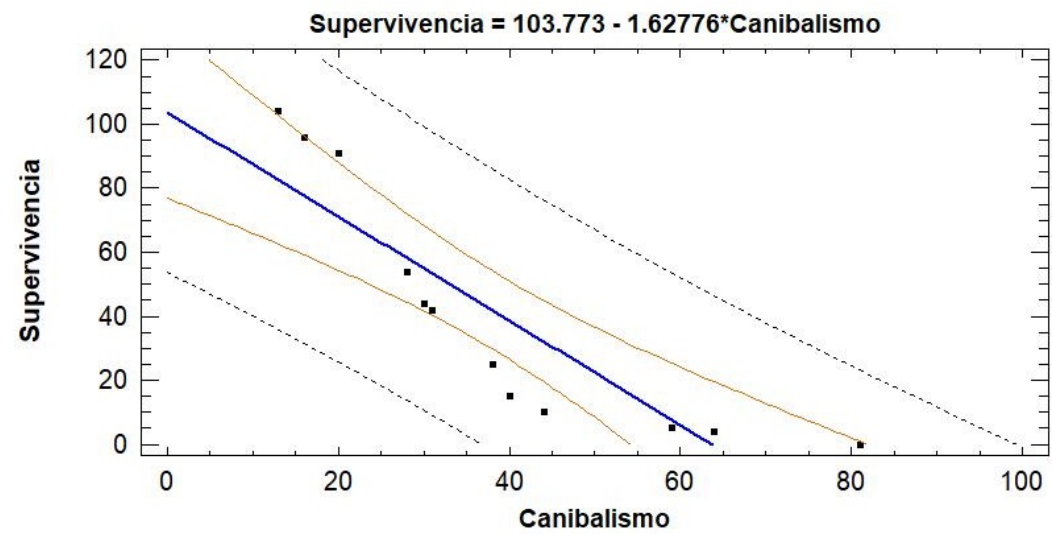

Figura 4. Modelo ajustado de regresión lineal simple entre supervivencia vs canibalismo en la larvicultura de Atractosteus tropicus. Ecuación del modelo: Surpervivencia $=103.78-1.63$ ${ }^{*}$ Canibalismo $(p<0.05)$.

puede deberse a que los organismos se alimentaron por 15 días más. La alta variabilidad en peso individual húmedo puede deberse a la mayor densidad de cultivo utilizada (4.3 individuos por $\mathrm{m}^{3}$ ), lo que incrementó la competencia por alimento los primeros días del cultivo, pudiendo ocasionar rezago y mayor depensación inicial. La depensación en densidades con mayor biomasa ha sido reportado en otras especies de peces carnívoros (Walters y Kitchell 2001), así como en especies omnívoras como Oreochromis niloticus (Gasca-Leyva et al. 2008).

En los peces alimentados con co-alimentación (Artemia-dieta formulada), el crecimiento en talla y peso fue superior $(p<0.05)$ al obtenido en otro experimento con dieta experimental a base de almi- dón de papa (Frías-Quintana et al. 2017), donde los organismos no lograron pesar más de $1 \mathrm{~g}$, además de tallas bajas entre 40 y $50 \mathrm{~mm}$, lo que refuerza el hecho de que la dieta experimental utilizada se adapta bien a los requerimientos nutricionales de $A$. tropicus. Los resultados sugieren que la dieta formulada incrementa el crecimiento de las larvas. Lo que coincide con Frías-Quintana et al. (2016), quienes utilizaron almidón de maíz como fuente de energía, así como harina de pescado y harina subproductos de ave como fuente de proteínas; obteniendo mayor crecimiento. La utilización de aceite de pescado mezclado con lecitina de soya como fuente de lípidos concuerda con la sugerencia de Huerta-Ortiz et al. (2009), quienes recomiendan usar aceite de pescado 
(sardina) mezclado con aceites de origen vegetal.

La supervivencia de todos los tratamientos fue menor al $50 \%$, resultados que son inferiores a la supervivencias entre 58 - 97\% reportada por FríasQuintana et al. (2016), con la misma dieta experimental, estas diferencias tal vez puedan deberse a que en el presente experimento la duración del cultivo fue mayor por 15 días, ya que se observó una mortalidad constante de organismos en los días finales del cultivo, así como un incremento de la agresividad. Así mismo, la diferencia de densidad en el diseño experimental pudo tener un efecto en la supervivencia, ya que mayor densidad supone una limitación espacial y mayor competencia por alimento. Pero la supervivencia obtenida es similar a los valores de FríasQuintana et al. (2017), quien evaluó la efectividad de utilizar diferentes cantidades de almidón de papa como fuente de energía, con supervivencias entre 16 $-28 \%$.

Sobre el canibalismo, Frías-Quintana et al. (2017) reporta valores mayores del $30 \%$, por lo que los valores observados en el presente estudio son bajos con excepción del porcentaje de canibalismo encontrado en el tratamiento T1. La alta mortalidad y canibalismo en dietas con almidón de papa, al compararlas con dietas con almidón de maíz, puede sugerir que incluir el almidón de maíz a la composición de la dieta inerte para $A$. tropicus reduce la incidencia de canibalismo, incrementando la supervivencia larvaria. Así como crecimiento superior, lo que indica que la dieta diseñada satisface los requerimientos nutricionales de las larvas.

Incluir alimento vivo como nauplio de Artemia salina es determinante para la supervivencia en el larvicultivo de $A$. tropicus, ya que se obtuvieron mejores porcentajes en los tratamientos de co-alimentación (CT $=15.56 \%$; T2 = 32.33\%). La importancia de la utilización de nauplios de Artemia salina coincide con el trabajo de Saenz de Rodrigáñez et al. (2018), quienes en su evaluación de dietas microencapsuladas para larvas de $A$. tropicus, encontraron una supervivencia mayor al $80 \%$ en los organismos alimentados con nauplios de Artemia sp. Los resultados en supervivencia también concuerdan con EscaleraVázquez et al. (2018), quienes reportan que la uti- lización de Artemia fransiscana como presa viva para las primeras semanas de alimentación incrementa la supervivencia y mejora la adaptación a la vida en cautiverio.

El canibalismo completo se observó los primeros días del cultivo, durante la adaptación al alimento inerte, como lo indica (Márquez-Couturier et al. 2015). Pero al igual que en la perca Perca fluviatilis la agresividad se observó después de la adaptación a la dieta comercial (Baras et al. 2003). En este aspecto, la alta correlación entre el canibalismo y la supervivencia $(r=-0.88, p<0.05)$, sugiere que utilizando una co-alimentación inicial de nauplio de Artemia sp. en conjunto con la dieta diseñada en función de la fisiología digestiva de la especie, reduce el canibalismo durante el larvicultivo, descartando la importancia de una adecuada biomasa de siembra inicial como un efecto mitigador del canibalismo.

Los resultados muestran alta correlación entre el canibalismo con la supervivencia, por lo que se requiere buscar soluciones para mitigar este comportamiento en la acuicultura de peces carnívoros, durante el periodo larval. Al igual que Naumowicz et al. (2017), se sugiere que controlar el canibalismo por medio de la domesticación de las especies, permitirá disminuir este fenómeno. Mejorar el pool genético en varias generaciones de reproductores, como sugieren Márquez-Couturier y VázquezNavarrete (2015), pudiera eliminar el carácter relacionado con la agresividad de la especie e incrementar la supervivencia. Finalmente, los resultados de este experimento sugieren que el éxito de la larvicultura depende del origen y calidad de los reproductores y las condiciones controladas de las zonas de desove en laboratorio, como se ha observado en el bagre europeo Siluris glanis (Król et al. 2014).

\section{CONCLUSIONES}

Se obtuvo mayor crecimiento y supervivencia, además de menor canibalismo en las larvas alimentadas utilizando una co-alimentación inicial con nauplio de Artemia salina y la dieta diseñada en función de la fisiología digestiva de $A$. tropicus, lo que sugiere que es una alternativa al uso de dieta comercial du- 
rante la larvicultura. Se requiere evaluar aspectos de biomasa de siembra inicial y presas utilizadas para disminuir el canibalismo de la especie.

\section{AGRADECIMIENTOS}

Al Consejo Nacional de Ciencia y Tecnología (CONACYT) por la beca de manutención para la realización de los estudios de posgrado.

\section{LITERATURA CITADA}

Arias-Rodríguez L, Paramo-Delgadillo S, Contreras-Sánchez WM, Álvarez-González CA (2009) Karyotype of the tropical gar Atractosteus tropicus Lepisosteiformes: Lepisosteidae) and chromosomal variation in their larval and adults. Revista de Biología Tropical 57: 529-539.

Baras E, Kestemont P, Mélard C (2003) Effect of stocking density on the dynamics of cannibalism in sibling larvae of Perca fluviatilis under controlled conditions. Aquaculture 219: 241-255.

Escalera-Vázquez LH, Domínguez-Domínguez O, Molina-Domínguez E, Sarma SS, Nandini S (2018) Determination of optimal prey for rearing the tropical gar Atractosteus tropicus (Lepisosteiformes: Lepisosteidae). Revista de Biología Tropical 66: 1018-1033.

Eslava-Eljaiek P, Wedler E, Serna-Macías D (2011) Caracterización y criterios de eclosión de quistes de Artemia sp. en la salina de pozos colorados (Santa Marta, Colombia). Intropica 6: 101-108.

Frías-Quintana CA, Álvarez-González CA, Márquez-Couturier G (2010) Design of microdiets for the larviculture of tropical gar Atractosteus tropicus, Gill 1863. Universidad y Ciencia 26: 265-282.

Frías-Quintana CA, Márquez-Couturier G, Álvarez-González CA, Tovar-Ramírez D, Nolasco-Soria H, GalavizEspinosa MA, et al. (2015) Development of digestive tract and enzyme activities during the early ontogeny of the tropical gar Atractosteus tropicus. Fish Physiology and Biochemistry 41: 1075-1091.

Frías-Quintana CA, Domínguez-Lorenzo J, Álvarez-González CA, Tovar-Ramírez D, Martínez-García R (2016) Using cornstarch in microparticulate diets for larvicultured tropical gar (Atractosteus tropicus). Fish Physiology and Biochemistry 42: 517-528.

Frías-Quintana CA, Álvarez-González CA, Tovar-Ramírez D, Martínez-García R, Camarillo-Coop S, Peña-Marín ES, et al. (2017) Use of potato starch in diets of tropical gar (Atractosteus tropicus, Gill 1863) larvae. Fishes 2(1): 3. Doi 10.3390/fishes2010003.

Gasca-Leyva E, Hernández JM, Veliov VM (2008) Optimal harvesting time in a size-heterogeneous population. Ecological Modelling 210: 161-168.

Guerrero-Zarate R, Álvarez-González CA, Olvera-Novoa MA, Perales-García N, Frías-Quintana CA, MartínezGarcía R, et al. (2013) Partial characterization of digestive proteases in tropical gar Atractosteus tropicus juveniles. Fish Physiology and Biochemistry 40: 1021-1029.

Huerta-Ortiz M, Álvarez-González CA, Márquez-Couturier G, Contreras-Sánchez WM, Civera-Cerecedo R, Goytortua-Bores E (2009) Sustitución total de aceite de pescado con aceite vegetal en larvas de pejelagarto Atractosteus tropicus. Kuxulkab 15: 51-58.

Król J, Flisiak W, Urbanowicz P, Ulikowski D (2014) Growth, cannibalism, and survival relations in larvae of european catfish, Siluris glanis (Actinopterygii: Siluriformes: Siluridae) - attempts to mitigate sibling cannibalism. Acta Ichthyologica et Piscatoria 44: 191-199.

Márquez-Couturier G, Vázquez-Navarrete CJ (2015) Estado del arte de la biología y cultivo de pejelagarto (Atractosteus tropicus). Agroproductividad 8: 44-51. 
Márquez-Couturier G, Vázquez-Navarrete CJ, Contreras-Sánchez WM, Álvarez-González CA (2015) Acuicultura Tropical Sustentable: Una estrategia para la producción y conservación del pejelagarto (Atractosteus tropicus) en Tabasco, México (2nd ed.), Villahermosa, Tabasco: Universidad Juárez Autónoma de Tabasco. $87 \mathrm{p}$.

Naumowicz K, Pajdak J, Terech-Majewska E, Szarek J (2017) Intracohort cannibalism and methods for its mitigation in cultured freshwater fish. Reviews in Fish Biology and Fisheries 27: 193-208.

Saenz de Rodrigáñez M, Aguilar-Tellez FV, Alarcón-López FJ, Pedrosa-Islas R, Peña-Marín ES, Martínez-García $\mathrm{R}$, et al. (2018) Alimentos microencapsulados para el cultivo de larvas de pejelagarto (Atractosteus tropicus). Revista de Biología Tropical 66: 1298-1313.

Walter C, Kitchell JF (2001) Cultivation/depensation effects on juvenile survival and recruitment: implications for the theory of fishing. Canadian Journal of Fisheries and Aquatic Sciences 58: 39-50. 
